\title{
Management of hypothyroidism in pregnancy
}

\author{
A Bohacikova*1, D Bullimore ${ }^{1}$, O Pereira ${ }^{2}$
}

1: Barnsley NHSFT, UK 2: Mid-Yorkshire Trust UK, *Presenting author

Introduction

Until $12^{\text {th }}$ week of gestation the foetus is entirely dependent on maternal thyroxine. Inadequate thyroxine replacement may lead to a substantially lower IQ of the offspring and also an increased miscarriage rate.

In the first trimester TSH is not an optimal monitoring marker of thyroid status as it is supressed by the rising HCG level. According to established clinical guidelines, in order to protect the foetus, FT4 should be maintained close to the upper limit of normal and TSH should be less than $2.5 \mathrm{mIU} / \mathrm{L}$ in the first trimester by appropriately adjusting the dose of levothyroxine by 8 weeks gestation.

We conducted an audit of pregnant hypothyroid females to answer the question "Has management of thyroid status met current standards?" and, if not, to decide how management should be improved.

\section{Method}

68 hypothyroid pregnant patients presenting in 2012 to Mid-Yorkshire Trust, UK, were identified using the Euroking database and hospital records. These formed the basis of the audit. Information extracted included gestation week when TFTs were taken, time to specialist referral and the appropriateness and the timing of any action taken with respect to the TFTs.
Results

In which gestation week were
TFTs taken?

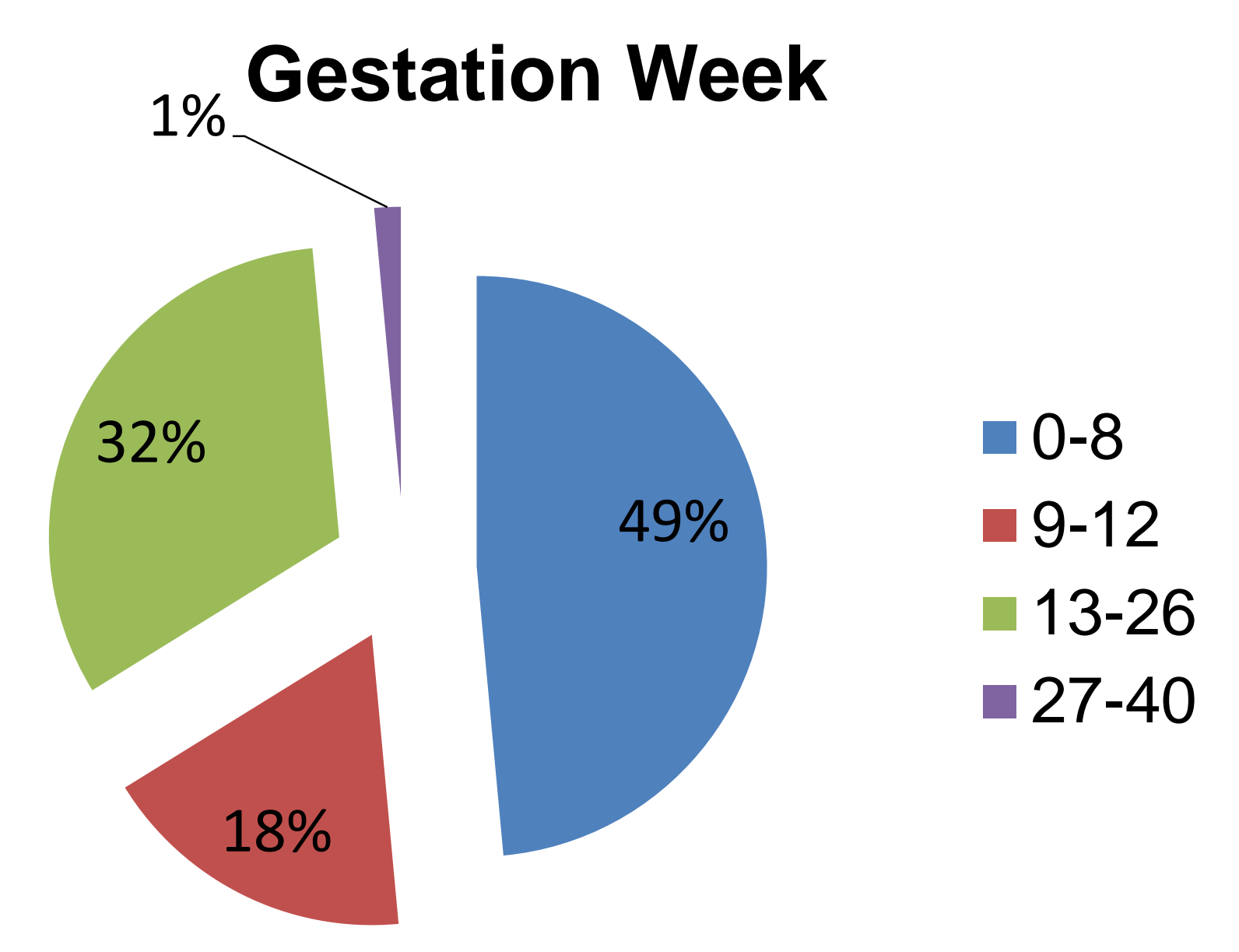

Only half (49\%) had samples taken in time to allow effective treatment change

How soon were they seen by a Specialist?

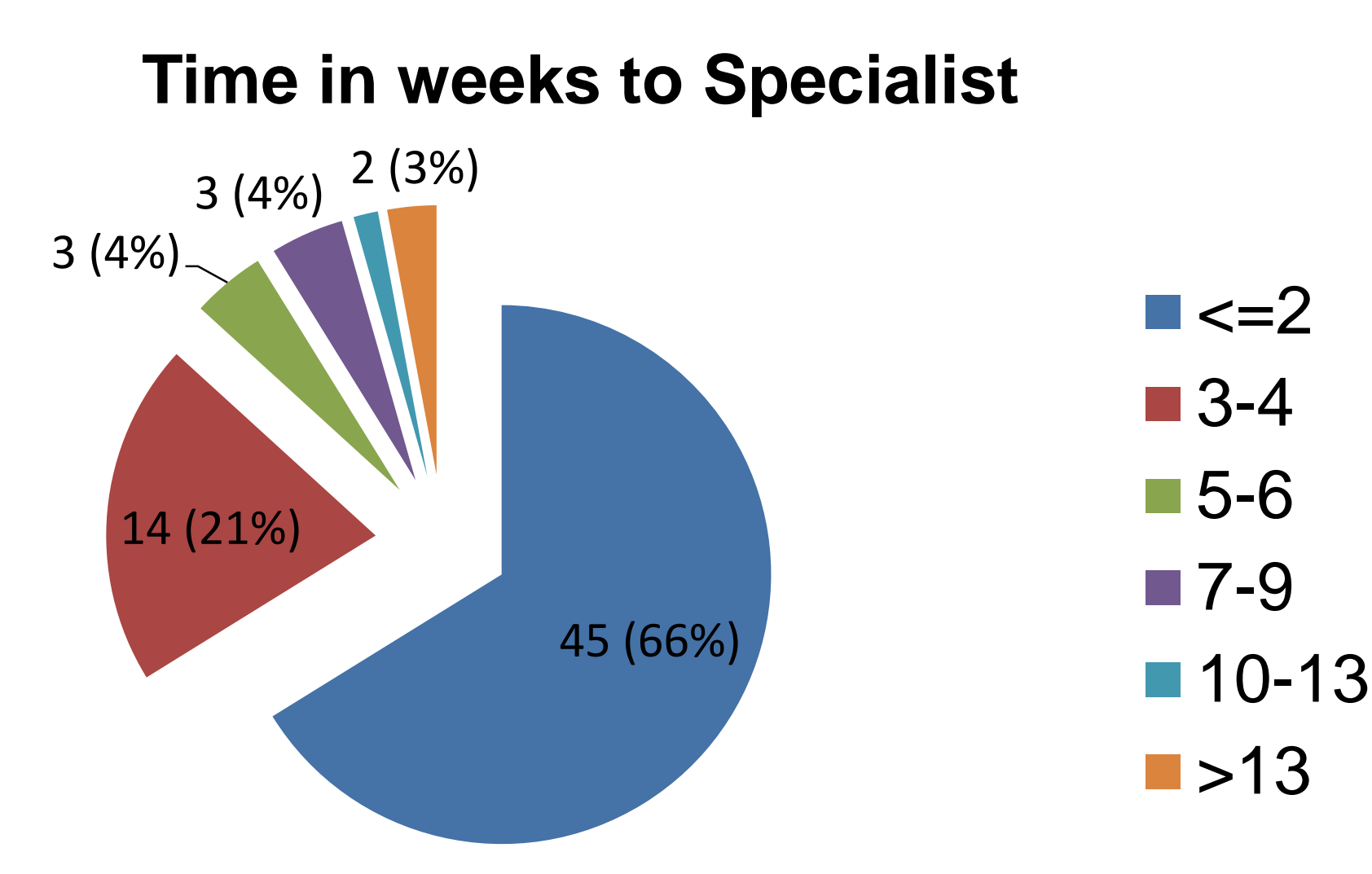

Two thirds were seen by Specialist within two weeks of referral

\section{What was the gestation when seen by Specialist?}

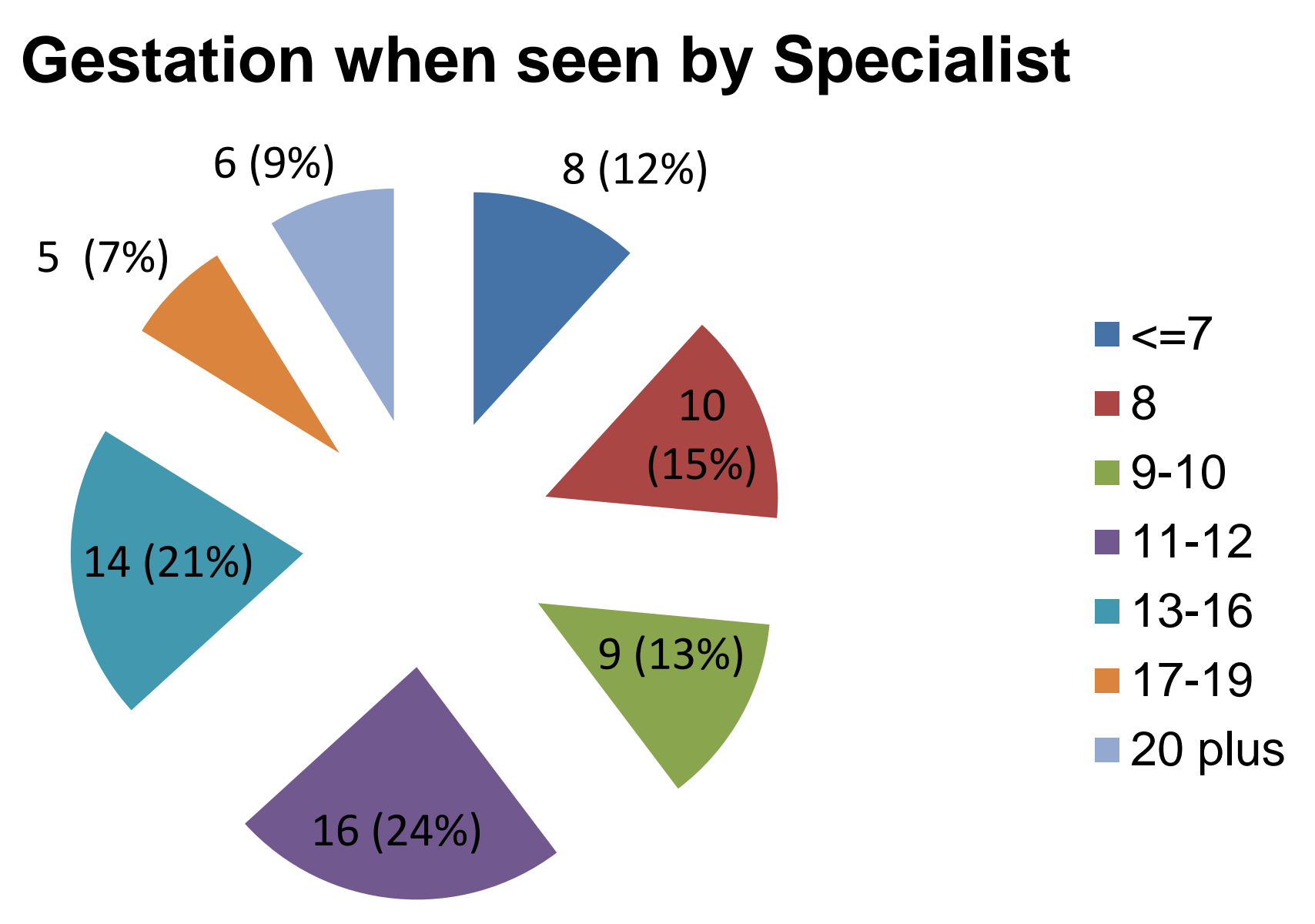

Only just over a quarter (27\%) were seen by specialist by $8 / 40$ gestation in time to implement effective change
Results (Continued)

What action was taken as a result of the TFTs?

Of 33/68 (49\%) who had TFTs at $<=8 / 40$ in time for any change in levothyroxine to be effective :

$18 / 33$ required no dose adjustment $9 / 33$ had dose levothyroxine increased $6 / 33$ should have had thyroxine dose increased

Of $23 / 68$ tested after $12 / 40,10 / 23$ should have had their dose of thyroxine increased.

\section{Discussion}

Only half the patients (49\%) had TFTs taken by 8 weeks gestation and only a quarter (27\%) where seen by the specialist in time to make effective change to levothyroxine dose if required. Two thirds saw the specialist within two weeks of referral. Of 33/68 checked by 8/40 18 patients required no dosage change and in 9 it was appropriately increased. However, in 6 patients who should have had a levothyroxine dose increase no change was made. Moreover review of results from 23/68 first tested after 12 weeks gestation suggested it was likely that $10(43 \%)$ should have had a dose increase in the first trimester and that the foetus may have been put at risk.

\section{Conclusion}

Currently accepted standards for monitoring and management of hypothyroidism in pregnancy were achieved in only $40 \%(27 / 68)$ of patients. Education of primary care physicians, midwives and patients is required so that they are aware of the need to undertake and act upon TFTs early in pregnancy or even before when conception is being contemplated. This will help to ensure a better outcome for both mother and baby.

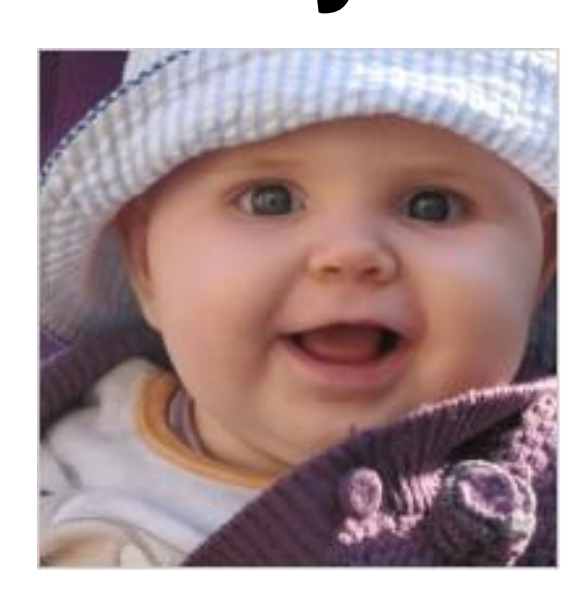

The audit needs repeating in a year to see if the situation has improved. 